\title{
REVISIÓN DE INVESTIGACIONES SOBRE LA ENSEÑANZA-APRENDIZAJE DE LOS CONCEPTOS CANTIDAD DE SUSTANCIA Y MOL
}

\author{
FURIÓ, CARLOS 1 , AZCONA, RAFAEL ${ }^{2}$ y GUISASOLA, JENARO 3 \\ ${ }^{1}$ Departamento de Didáctica de las Ciencias Experimentales y Sociales. Universitat de València \\ 2 Instituto de Enseñanza Secundaria Talaia. Hondarribia \\ ${ }^{3}$ Departamento de Física Aplicada I. Universidad del País Vasco
}

\begin{abstract}
Resumen. El objeto de este trabajo es revisar la situación actual de las investigaciones didácticas realizadas en el campo de la química sobre los conceptos de cantidad de sustancia y de mol. La revisión está organizada en cuatro apartados: dificultades de aprendizaje, dificultades de enseñanza, controversias sobre la magnitud «cantidad de sustancia» y estrategias de enseñanza. Palabras clave. Dificultades de aprendizaje, cantidad de sustancia y mol, dificultades de enseñanza, estrategias de enseñanza, revisión de investigaciones.
\end{abstract}

Summary. The aim of this paper is to review the present situation of «amount of substance» and mol concepts research in Chemical Education. We organise the review in four fields: learning difficulties, teaching difficulties, controversies about the magnitude of «amount of substance» and teaching strategies.

Keywords. Learning difficulties, amount of substance and mole, teaching difficulties, strategies of instruction, review of research.

\section{INTRODUCCIÓN}

La abundante literatura en el campo de la didáctica de la química de las últimas décadas ha puesto de manifiesto la existencia de un gran número de trabajos en torno al problema de la enseñanza-aprendizaje del concepto de mol (Dierks, 1981; Cervellati et al., 1982; Lazonby et al., 1985; Nelson, 1991; Tüllberg et al., 1994; Staver y Lumpe, 1995). En este sentido hay trabajos que abordan este problema didáctico desde diversas vertientes: las percepciones de los estudiantes, las percepciones de los profesores, las nuevas perspectivas didácticas planteadas a la luz de la psicología del aprendizaje, el punto de vista histórico y fílosófico sobre el origen y la evolución de los conceptos, los prerrequisitos necesarios para el aprendizaje de estos conceptos, etc.

El punto de partida del problema que nos preocupa se sitúa en las dificultades de aprendizaje que plantea a los estudiantes el concepto de mol, tal y como ha sido puesto de manifiesto de forma reiterada por la investigación didáctica al respecto, durante las dos últimas décadas. En este sentido se ha llegado a afirmar (Kolb, 1978) que «there is probably no concept in the entire first-year chemistry course more important for students to understand than the mole, and one of the main reasons the mole concept is so essential in the study of chemistry is stoichiometry».

Por otra parte, a partir de la adopción, por la comunidad científica internacional (Mills et al., 1993), de la «cantidad de sustancia» como una de las siete magnitudes fundamentales de la que el mol es su unidad, el problema adquiere otra dimensión más amplia. En este sentido han empezado a aparecer en la literatura algunos trabajos sobre la magnitud «cantidad de sustancia» en los que se 
pone de manifiesto que ésta no tiene un claro significado para los estudiantes y el profesorado (Furió et al., 1993; Tüllberg et al., 1994).

Así mismo, Gabel y Bunce (1994) plantean que el problema didáctico ya no se limita a las dificultades de los estudiantes sino que la causa del mismo está en la enseñanza:

«[...] Because the mole is a concept devised by scientists to aid in chemical calculations, students' errors or nonconceptions could hardly be called intuitive conceptions. They arise because of insufficient instruction or inappropiate teaching strategies.»

De acuerdo con lo anterior, podemos decir que existe en la actualidad una profunda preocupación por parte del profesorado en torno al tema. La disparidad de puntos de vista se puede observar en diferentes estudios. La revisión sobre la introducción y la utilización del concepto de mol que llevó a cabo Dierks (1981) incluye abundantes referencias bibliográficas posteriores a 1960. Otros estudios se han centrado en el análisis del contenido de libros de texto respecto a cómo se introduce el concepto de mol (De Berg, 1986a).

En el presente artículo se trata de realizar una revisión bibliográfica de las investigaciones que se centran en torno a las dificultades de aprendizaje de los conceptos de mol y cantidad de sustancia y en las alternativas didácticas que se ofrecen desde distintas perspectivas. Así mismo, incluiremos aquellos estudios que tratan sobre los prerrequisitos que ha de saber el estudiante para facilitar la construcción de sus significados y sobre su aplicación en los cálculos estequiométricos. Con ello esperamos contribuir a mostrar la verdadera dimensión del problema de la enseñanza-aprendizaje de estos conceptos.

\section{Dificultades de aprendizaje en torno a los conceptos de cantidad de sustancia y de mol}

Son numerosos los trabajos catalogados en la literatura didáctica sobre cuáles son las principales dificultades de aprendizaje del concepto de mol. Parece que existe un consenso en la investigación didáctica actual en que los estudiantes carecen de una concepción científica del mol (Gabel y Bunce, 1994). En este sentido, mediante una encuesta acerca del concepto de mol dirigida a una amplia población estudiantil desde educación secundaria (16 años) hasta primer curso universitario (19 años), García y otros (1990) refieren elevados porcentajes de respuestas erróneas, que discrepan de la definición de la IUPAC, y concluyen que hay un aprendizaje superficial del concepto.

En el trabajo de Dierks (1981) citado anteriormente, tomando como referencia las definiciones de la IUPAC sobre el concepto de mol (1958 y 1967), se indica que solamente pueden hacerse suposiciones provisionales sobre los efectos producidos por las diferentes definiciones del concepto de mol y apunta, como principales dificultades de aprendizaje, el carácter abstracto de la expresión cantidad de sustancia y la atribución de diversos significados a la palabra mol. El autor explica que en la amplia revisión bibliográfica realizada ha encontrado que al mol se le atribuye, entre otros, el significado de unidad individual de masa, de porción de sustancia y de número de partículas (número de Avogadro). Así mismo, el trabajo pone un énfasis especial en la necesidad de clarificar el significado de la magnitud «cantidad de sustancia» de la cual deriva el mol como unidad.

De acuerdo con la revisión anterior, se ha comprobado que las principales concepciones erróneas de los estudiantes de 15 años eran identificar el mol con una masa o con un cierto número de partículas de gas, o bien considerar que el mol es una propiedad de una molécula (Novick y Menis 1976).

En otra investigación realizada con una amplia muestra de estudiantes de educación secundaria, Cervellati y otros (1982) mostraron que éstos percibían el mol como una masa, no lo utilizaban como unidad de «cantidad de sustancia» y relacionaron estas deficiencias con las dificultades de los estudiantes en la resolución de problemas estequiométricos. Apuntaron, acertadamente, que las posibles causas de esta situación eran debidas a la enseñanza en particular: el inadecuado contenido del currículo, la metodología de enseñanza utilizada, el sistema de evaluación y la formación del profesorado. Con el fin de superar estas dificultades sugieren, entre otras, la necesidad de revisar los métodos de enseñanza.

Con objeto de averiguar si la causa de las grandes dificultades que tenían los estudiantes de enseñanza secundaria, al resolver problemas, era el concepto de mol o bien era el trabajar con masa, volumen y partículas, Gabel y Sherwood (1984) confeccionaron un test sobre el concepto de mol, en el que sustituyeron los nombres químicos de las sustancias por otros más familiares como azúcar y naranjas, y utilizando docenas en lugar de moles. Los resultados de este trabajo mostraban que la dificultad en la resolución de problemas era debida, probablemente, a la utilización del término mol y de otros términos no familiares, más que a la falta de comprensión del volumen, la masa y el conjunto de partículas.

Por otra parte, Krishnan y Howe (1994) encontraron que los estudiantes de segundo año de enseñanza secundaria y los de un curso de introducción a la química universitaria en Estados Unidos tenían una deficiente comprensión del significado del término entidades independientes en la definición de mol. En este sentido también constataron que los estudiantes creen a menudo que el mol tiene que ver solamente con moléculas y no con átomos, y que el término cantidad en la definición de mol tiene significado de «masa constante».

Staver y Lumpe (1995) investigaron la comprensión del concepto de mol por los estudiantes de bachillerato y su utilización en la resolución de problemas, comprobando que algunos lo identifican con número de partículas y otros, con masa en gramos, a pesar de haberlo definido 
previamente de acuerdo con el sistema internacional. También refieren estos autores que los estudiantes tienen los siguientes impedimentos: incapacidad para transferir significados entre los niveles macro y micro (atómico/ molecular) cuando se resuelven problemas, insuficiente comprensión de los conceptos, utilización de algoritmos, reglas u otra información memorizada. Y apuntan, a modo de hipótesis, la preconcepción en los estudiantes de que el gramo y la unidad de masa atómica son equivalentes.

Furió y otros (1993) tratan de detectar las representaciones mentales de los estudiantes sobre la magnitud «cantidad de sustancia» y analizar en qué medida la enseñanza habitual de la química en educación secundaria contribuye a que los estudiantes tengan una visión más significativa de la misma. Los resultados obtenidos parecen indicar que los estudiantes, al terminar sus estudios de secundaria, no han cambiado prácticamente sus ideas macroscópicas sobre la expresión cantidad de sustancia, que identifican con la masa $\mathrm{y}$, en menor medida, con el volumen.

Existe una gran cantidad de trabajos que hacen referencia a las dificultades detectadas en los estudiantes al aplicar el concepto de mol en la utilización de cálculos estequiométricos. A continuación haremos referencia a los más destacados en la bibliografía.

Duncan y Johnstone (1973) detectaron una serie de dificultades de los estudiantes cuando la proporción estequiométrica (de «cantidad de sustancia») en una reacción no es 1:1. Así mismo, encontraron dificultades en la resolución de ejercicios sobre concentración de disoluciones al no tener en cuenta que, al diluir la disolución de una sustancia en agua, se altera el volumen de la disolución y, en consecuencia, no utilizan la expresión $N_{1} . V_{2}=N_{2} . V_{2}$ para calcular la nueva concentración (es decir, sin darsecuenta, mantienen constante el volumen).

Al estudiar el problema en torno a conceptos volumétricos, Vincent (1981) encontró tres tipos principales de preconcepciones: $a$ ) la disolución era considerada como una sustancia simple; $b$ ) la relación 1:1 en moles, en una neutralización, era igualada a la relación 1:1 en volumen, esto es, se da una fijación funcional de la concentración de las disoluciones de ácido y base; y c) los conceptos de cantidad y concentración eran confundidos entre sí.

En otra investigación realizada con una amplísima muestra (más de 6.000 estudiantes) de enseñanza secundaria para averiguar cuáles eran las estrategias utilizadas en estequiometría, Schmidt (1990) apunta que, cuando los estudiantes hacen cálculos estequiométricos, tienden a creer que la relación del número de moléculas es idéntica que la relación de masas en una reacción química, o bien que la relación de masas molares de las especies reaccionantes es igual que su relación de masas. Por lo que respecta a los cálculos sobre fórmulas químicas, también se indica que los estudiantes no suelen tener en cuenta que átomos de elementos diferentes tienen diferentes masas atómicas. En un estudio realizado posteriormente por el propio Schmidt (1994) con objeto de profundizar en el conocimiento de las estrategias utilizadas en la resolución de sencillos ejercicios de cálculo estequiométrico, destaca que los estudiantes evitan el cálculo directo de cantidades expresadas en moles, de lo cual deduce el autor que esto puede ser debido a que encuentran dificultades con el concepto de mol y, por otra parte, pone de manifiesto que los estudiantes no adoptan las estrategias de razonamiento para las que fueron preparados sino sus propios métodos.

En torno a esta última cuestión y en un trabajo realizado con anterioridad sobre cálculos estequiométricos en una volumetría redox, Frazer y Servant (1986a) encontraron que ningún estudiante, de una muestra de más de 200 que habían obtenido el certificado general de educación nivel avanzado exigido en Gran Bretaña para acceder a la universidad o centros superiores, utilizaba el método experto de resolución que implicaba el cálculo y manejo de «cantidades de sustancia». En una continuación del trabajo anterior, Frazer y Servant (1986b) presentan los resultados de una investigación realizada en laboratorios industriales, médicos y de tecnología del metal, en la que se muestra la amplia utilización de los equivalentes y de las normalidades como forma de expresar la concentración de las disoluciones, y de aquí infieren que el concepto de equivalente permite correlacionar datos empíricos sin recurrir a una teoría abstracta (en alusión a la teoría atómico-molecular). El mantenimiento de estos conceptos (equivalente, normalidad) en la química analítica utilizada en profesiones liberales puede explicarse debido a su sencillez y al carácter pragmático y funcional que representan. Sin embargo, suponen una asunción acrítica de conceptos de base empírica pero correspondientes al cuerpo preteórico existente en los siglos XVIII, XIX. La persistencia de conceptos como equivalente y normalidad en la enseñanza podría explicarse por la ausencia de reflexión sobre la construcción de los mismos.

Entre las investigaciones realizadas con objeto de conocer y superar las dificultades de aprendizaje del concepto de mol, algunas abordaban el problema desde un enfoque de la psicología del aprendizaje, poniendo un énfasis especial en las dificultades intrínsecas de comprensión del propio concepto debido a su no-adecuación a los niveles piagetianos de desarrollo cognoscitivo de los estudiantes (Goodstein y Howe, 1978). En este caso, algunos autores afirman, expresamente, que las dificultades no residen en la enseñanza o en que los estudiantes no se esfuercen en aprender, sino que son debidas a que son pocos los estudiantes que han alcanzado el nivel intelectual de las operaciones formales, como describe Piaget. Este nivel sería necesario en el aprendizaje de la química y, en particular, la comprensión del significado del concepto de mol (Herron, 1975; Shayer y Adey, 1984).

Goodstein y Howe (1978) afirman que los estudiantes de nivel concreto no pueden aprender conceptos que requieren pensamiento operacional formal avanzado. Esta línea de investigación que parte de asignar un carácter 
relevante a los procesos piagetianos de razonamiento formal en la enseñanza de las ciencias se refiere al conflicto que supone la existencia de un desfase (distancia cognitiva) entre las estructuras lógicas de las disciplinas como la física y la química y la estructura cognitiva de los estudiantes. Diversos autores afirman que puede mejorarse el aprendizaje de conceptos basados en operacionales formales, utilizando modelos concretos durante el proceso de enseñanza, o bien proponen posponer la enseñanza que requiere este tipo de operaciones en el caso de comprobarse que los estudiantes no las han adquirido. En este mismo sentido, apuntan que incluso los estudiantes de secundaria entran en la universidad sin haber alcanzado el estadio piagetiano operacional formal (Niaz, 1985, 1987). Refiriéndose a los resultados obtenidos en los cursos de introducción de física, química y matemáticas por estudiantes venezolanos en la universidad, Niaz (1985) afirma que un $75 \%$, aproximadamente tiene que repetir curso y que un $50 \%$ del contenido de estos cursos requiere razonamiento operacional formal. En efecto, los resultados obtenidos por este autor, al pasar un test de razonamiento operacional formal a una amplia muestra de estudiantes de primer curso de universidad, corroboran que más del $80 \%$ de los estudiantes operaban en el nivel operacional concreto y que sólo un 3,5\% se situaban en la categoría formal. En el trabajo se sugieren como soluciones a este problema: a) enseñar ciencias y matemáticas a estudiantes novicios en el nivel operacional concreto; y $b$ ) desarrollar el razonamiento operacional formal como uno de los principales objetivos.

Siguiendo con la línea de investigación neopiagetiana, otro tipo de trabajos (Pascual-Leone y Goodman 1979; Johnstone y El-Banna, 1986; Niaz, 1988 y 1989) ha centrado la atención en la dificultad que supone la cantidad de procesamiento de la información que ha de realizar el sujeto debido a la tarea que ha de resolver (denominado demanda $M$ por Pascual-Leone). En este sentido, Johnstone y El-Banna (1986) resaltaron la gran disminución en el rendimiento de los estudiantes que se obtenía al resolver problemas sobre el mol, cuando la exigencia cognitiva del problema (demanda $M$ ) es mayor que la capacidad de aquéllos para resolverlo. Niaz (1988 y 1989) ha mostrado, en coherencia con los resultados anteriores, que el rendimiento en la resolución de problemas de química de diferentes tópicos disminuye a medida que aumenta la demanda $M$, haciendo hincapié en la necesidad de hacer disminuir el nivel de demanda $M$ de un problema sin cambiar su estructura lógica.

Este planteamiento centra exclusivamente la atención en el proceso de aprendizaje de los estudiantes, obviando las influencias de tipo metodológico en las que más directamente puede incidir el profesorado. En este sentido, diversos autores (Driver, 1986) se refieren a que los resultados de varias investigaciones realizadas por psicólogos y educadores muestran que las estructuras lógicas que utilizan los estudiantes dependen en gran medida del contexto de la tarea, poniendo así en cuestión la idea de etapas coherentes de desarrollo cognoscitivo. Vigotsky (1989), por otra parte, indica que las tareas a resolver por los estudiantes han de relacionarse con sus capacidades, de modo que requiera acciones intelectuales y operativas pertenecientes a la «zona de desarrollo potencial próximo». Según este autor, existe un nivel evolutivo potencial en el proceso de aprendizaje, que se establece como resultado de procesos cognitivos que se encuentran en proceso de maduración y que se manifiestan a través de acciones intelectuales y operativas que un individuo puede desarrollar bajo la guía de un experto o en colaboración con otro compañero más capaz. Este nivel define una «zona de desarrollo potencial» a la que el aprendiz puede acceder si es convenientemente guiado.

Las objeciones anteriores han dado lugar a una línea de investigación más centrada en la ayuda que puede proporcionar la enseñanza y, en particular, en los métodos que emplean los profesores en sus clases. Es decir, si la distancia cognitiva es grande, y como tal se reconoce en el caso del concepto de $\mathrm{mol}$, la enseñanza ha de tratar de poner más medios para ayudar al aprendiz a disminuir aquella distancia. Así pues, pasaremos a revisar este tipo de trabajos en el siguiente apartado.

\section{PENSAMIENTO DEL PROFESORADO SOBRE LOS CONCEPTOS DE MOL Y DE CANTIDAD DE SUSTANCIA}

Tal y como hemos visto hasta aquí, el problema de aprendizaje inicialmente planteado para estudiar las causas de las equivocaciones de los estudiantes respecto al concepto de mol ha llevado consigo una investigación paralela sobre la forma de mejorar su enseñanza y sobre el significado atribuido por el profesorado a dichos conceptos. En este sentido, Hawthorne (1973) realizó el análisis de un centenar de textos de química desde 1891 hasta 1970, encontrando una relación creciente entre la idea del mol (introducido por Ostwald a principios del siglo xx) y el número de Avogadro.

A través del análisis de una veintena de libros de texto, Staver y Lumpe (1993) encontraron que predominan dos formas de definir el mol. En ambas, el requerimiento cognitivo es muy alto debido a su relación con el número de Avogadro de partículas y a que compara la masa de sustancia contenida en el mol con $12 \mathrm{~g}$ del isótopo ${ }^{12} \mathrm{C}$. Por otra parte, estos autores apuntan que en casi todos los textos se presenta el mol en relación con el problema de descubrir un modo de contar partículas que son demasiado pequeñas para ser pesadas directamente. Así mismo, se hace énfasis en la necesidad de utilizar analogías con conceptos familiares a la hora de introducir el concepto.

Strömdahl y otros (1994) han realizado un interesante estudio sobre las concepciones de mol que tienen los profesores, en el que obtuvieron que sólo un 10,7\% identificaba el mol como unidad de la magnitud «cantidad de sustancia», siendo las opciones mayoritarias las identificaciones con número de Avogadro (60,7 \%) y con masa $(25 \%)$. Estos autores concluyen que las concepciones sobre mol de los estudiantes son consecuencia de las que tienen los profesores y que estas visiones 
difieren de la expresada por la comunidad científica en el sistema internacional. En un trabajo complementario posterior (Tullberg et al., 1994), se pone de manifiesto que los conceptos que causan más problemas a los estudiantes son aquéllos que están ausentes en la enseñanza y se dan por sabidos como, por ejemplo, la diferenciación entre masa molar y masa atómica o molecular. Estos autores indican que el profesorado está muy condicionado por sus propias concepciones sobre el mol, haciéndose necesario conocer todas las implicaciones de la definición del sistema internacional para que el profesor se dé cuenta de sus propias concepciones.

En un amplio análisis crítico de la enseñanza de los conceptos de cantidad de sustancia y de mol (Azcona, 1997, Furió et al., 1999, Azcona, 2001), se pusieron de manifiesto, entre otras, las siguientes conclusiones:

- El profesorado desconoce el carácter evolutivo del concepto de mol desde su contexto original de indagación hasta su significado actual acorde con las recomendaciones de la comunidad científica internacional. Así mismo, la relativamente reciente introducción de la «cantidad de sustancia» (1961) como magnitud fundamental puede explicar la ausencia de este concepto en la mayoría de los programas actuales de enseñanza de la química.

- El concepto de mol se introduce en la mayoría de los textos de química, atribuyéndole equivocadamente significados de masa química o de número de entidades elementales. Esta visión errónea también se mantiene en autores y publicaciones de reconocido prestigio y en el profesorado coincidiendo con los resultados de Strömdahl y otros (1994). Estas representaciones mentales equivocadas se transmiten en la enseñanza y persisten en los estudiantes.

- La presentación del concepto de mol en las programaciones de la enseñanza habitual muestra una visión distorsionada de la ciencia y del trabajo científico, sin explicitar cuál es el problema actual que trata de resolver el concepto de mol. Al propio tiempo, el profesorado no tiene en cuenta la evolución conceptual experimentada por el mol, al pasar de un marco equivalentista en el que fue ideado originalmente a un marco teórico distinto como es el atomista aceptado actualmente.

\section{CONTROVERSIAS SOBRE LA TERMINOLO- GIA DE LA CANTIDAD DE SUSTANCIA, EL SIGNIFICADO DE MOL Y OTROS CONCEP- TOS RELACIONADOS}

Al margen de los trabajos de investigación a los que hemos hecho referencia, existen abundantes referencias bibliográficas que ponen de manifiesto que el problema que estamos considerando supera dicho ámbito y preocupa sobremanera a la comunidad científica. En estas referencias hay opiniones discrepantes respecto a los significados atribuidos a los conceptos de cantidad de sustancia y mol. Existe una gran variedad de opiniones $y$, en determinados momentos, las revistas sobre investigación en educación científica se han convertido en foros de debate sobre auténticas polémicas originadas en torno al significado de estos conceptos. Resumimos a continuación los debates aparecidos en la bibliografía.

\section{En relación con la terminología de la expresión can- tidad de sustancia}

Algunos trabajos apuntan que no es necesaria esta magnitud. McGlashan (1977) justifica que no se necesita epistemológicamente la magnitud «cantidad de sustancia» $(n)$, puesto que ésta sirve para contar, indirectamente, partículas o entidades elementales, y para ello la teoría atómica ya maneja el concepto de cantidad de entidades elementales $(N)$ aunque de entrada no se pueda medir directamente. En este sentido, Nelson (1991 y 1994) hace referencia explícita a la complicación que supone la introducción de la «cantidad de sustancia» en la realización de cálculos estequiométricos.

Otros trabajos muestran desacuerdo con la denominación cantidad de sustancia en el sistema internacional (Clayton, 1981; Lee, R.E., 1982; Clayton, 1983; McManus, 1983) o con la consideración del mol como unidad de la magnitud «cantidad de sustancia» (Lee, S., 1961; Lee, R.E., 1982). Se trata de mostrar el carácter confuso del nombre (McManus, 1982) y se hacen propuestas o se utilizan otros nombres para designar la magnitud. Veamos algunos ejemplos:

- metromoriance o chemiance, en referencia a medir partículas pequeñas (Gorin, 1982);

- molar amount (Copley, 1961; Kohman, 1987);

- moleage (Clayton, 1981, 1982). This denomination is consistent with the practice of describing a length in mile units as a mileage;

- ontcount to refer to a large counting unit of any form of elementary entities (Forbes, 1977, 1978b, 1982);

- particulality because matter is composed of particles (Tykodi, 1983);

- chemical amount: the amount of matter that contains a number of chemically defined particles (Gorin, 1982, 1983, 1984; Nelson, 1989; Mills, 1989; Hoppé, 1990; Nelson, 1991);

- numerousness o numerosity to state that the matter handled by chemists is a collection of extremely numerous entities (Rocha-Filho, 1990);

- entity-count (Forbes 1991) to refer to the operation of counting elementary entities;

- chemount: synthetic expression of «chemical amount» (Woods, 1991).

Sin embargo, trabajos más recientes (Hoppé, 1991) critican la propuesta de nuevos nombres para denominar la 
«cantidad de sustancia» por considerar que contribuyen a aumentar la confusión existente. Verdú (1993) resalta que esta magnitud es la única que no depende de las condiciones del sistema (es invariable) si éste no cambia de entidad elemental. Varios trabajos apuntan que se necesita una palabra para designar «por cantidad de sustancia» y se sugiere la palabra propia -por ejemplo, la «energía propia» de una sustancia es de tantos joule por mol mejor que decir la «energía molar» es de tantos joule por mol (Guggenheim, 1961) o la palabra cardinal (Cohen, 1961a). Así mismo, Forbes (1976, 1978a) apunta la conveniencia de una unidad a nivel atómico para «cantidad de sustancia» proponiendo la llamada unidad de sustancia ordinaria (osu), definida como la cantidad de sustancia o el ont-count de un sistema que contiene una entidad elemental.

En resumen, parece que la magnitud «cantidad de sustancia» tiene un nombre ambiguo debido a que cantidad tiene diversos significados y a que puede utilizarse para medir entidades que no son sustancias (Gorin, 1994). Sin embargo, parece existir un consenso en que se trata de una magnitud fundamental, aunque queda como cuestión importante a resolver la búsqueda de un sinónimo de «cantidad de sustancia». En este mismo sentido, y en el campo de la física, la denominación cantidad de electricidad se utilizó inicialmente para nombrar al número de «masas eléctricas» hasta que se encontró un sinónimo diferente a masa y se le denominó más propiamente carga eléctrica. Recientemente la IUPAC ha adoptado (Mills et al., 1993) la denominación alternativa cantidad química. (Creemos que la palabra cantidad sigue teniendo un significado asociado a número, que puede dificultar su interpretación como magnitud.)

\section{En relación con el significado del mol}

Una serie de trabajos muestran su desacuerdo con el significado atribuido a la magnitud «cantidad de sustancia» y a su unidad, el mol. Así, McGlashan (1977) manifiesta que no es necesaria esta unidad y aboga por abandonar progresivamente las magnitudes molares utilizando en su lugar magnitudes atómicas o moleculares. Se apunta la necesidad de redefinir el concepto de mol como número o se atribuye al mol significado numérico (Lee, S., 1961; Bieber, 1961; Cohen, 1961b; McManus, 1982). Clayton $(1981,1982,1983)$ muestra desacuerdo con la definición de mol de la IUPAC e indica que el $\mathrm{mol}$ no es una unidad básica, sino una variedad de unidades -moles, moléculas, gramos o metros cúbicos-. Así mismo, Brown (1991) afirma que el mol indica la «cantidad de sustancia» en tres modos diferentes: masa, volumen y número de entidades. Nelson (1991) muestra desacuerdo con que el mol sea considerado como una unidad para contar y Ainley (1991) apunta ventajas pedagógicas al considerar que un mol es una porción de sustancia admitida como masa.

Otros trabajos se centran en la necesidad de redefinir el concepto de mol y de clarificar su significado. En este sentido, se muestra en desacuerdo con que el mol sea considerado como la docena de los químicos - «ya que es la constante de Avogadro lo que es la constante de los químicos y no el mol» (Hudson 1976) - o como un número (Forbes, 1982; Mills, 1989; Hoppé, 1990; Nelson, 1991; Spurgin, 1992; Verdú, 1993). Se hace referencia al mol o a la cantidad de sustancia como una forma de contar (MacDonald, 1984; Fernández, 1989). Se apunta como error identificar el mol como masa o como volumen molar (Verdú, 1993) y se identifica el mol o la «cantidad de sustancia» con un colectivo o con un número de partículas (Verdú, 1993; Foy, 1961; Lee, 1982; Clayton, 1982).

Por otra parte, un número importante de referencias bibliográficas muestra de modo explícito el acuerdo con la definición actual del sistema internacional (Gorin, 1983; Caamaño, 1983; Caamaño et al., 1983; Smith, 1984; Ramette, 1988; Spurgin, 1992; Verdú, 1993). Así, Allsop (1977) apunta la ventaja de clarificación y simplificación que implica la aplicación del concepto de $\mathrm{mol}$ a la electroquímica al permitir reinterpretar la segunda ley de Faraday sin la inclusión de equivalentes electroquímicos. Así mismo, diversos autores hacen referencias al origen histórico del concepto (Gorin, 1983; Nelson, 1991) y se recomienda la necesidad de una introducción histórica del mol (Caamaño et al., 1983).

\section{Sobre el significado de otros conceptos relacionados con el mol}

Existen abundantes referencias bibliográficas en las que se muestra una diversidad de puntos de vista sobre el significado de determinados conceptos tales como la constante de Avogadro, la masa molar o la concentración, relacionados con la magnitud «cantidad de sustancia». A continuación se hace referencia a alguno de estos trabajos.

En relación con la constante de Avogadro, Forbes (1978a) considera inadecuada la denominación propuesta por la IUPAC. Otros autores hacen referencia a la necesidad de diferenciar el número y la constante de Avogadro (Verdú, 1993), considerando también la dificultad que supone la introducción de un número tan enorme (el número de Avogadro), que supera la imaginación de los estudiantes (Steiner, 1986).

En relación con la masa molar, Retherford (1978) propone la utilización de la expresión masa atómica - gramo en lugar de la masa molar. Por otra parte, Copley (1961) define masa minimolar de un elemento como «la menor masa de un elemento que puede encontrarse en las masas molares de sus compuestos». En época más reciente, se propone la utilización de la masa molar como la masa en gramos de un mol de una sustancia (Masson, 1993) y se considera que la masa molar es una constante física o química como la densidad (Ten Hoor, 1993).

En relación con la forma de expresar la concentración de las disoluciones, algunos autores abogan, de acuerdo con la IUPAC, por la no-utilización del adjetivo molar para referirse a la concentración de una disolución (Gorin, 1985) o indican que la molaridad no es una 
magnitud molar debido a que el término molar significa «por unidad de cantidad de sustancia» (Verdú 1993). Sin embargo, otros autores se plantean la utilización de términos distintos a los propuestos por la IUPAC para denominar la concentración de las disoluciones: concentración molar (Kohman, 1987), molevity en lugar de molaridad, para expresar la concentración de cantidad por litro de disolución o molamity en lugar de molalidad, para expresar la «cantidad por kilogramo de disolvente» (Elsworth, 1990), con cuyas expresiones otros autores muestran su desacuerdo (Mills, 1990).

\section{ESTRATEGIAS DE ENSEÑANZA DE LOS CONCEPTOS DE CANTIDAD DE SUSTAN- CIA Y DE MOL}

Al igual que en el caso del aprendizaje, también existen abundantes trabajos que han investigado el problema desde el punto de vista de la enseñanza del concepto de mol. Sin embargo, el número de trabajos desciende notablemente cuando se trata de la magnitud «cantidad de sustancia». Aunque todos ellos tienen en común las dificultades de aprendizaje que plantean dichos conceptos, podemos apuntar cuatro tipos principales de orientación de estos trabajos, si bien algunos de ellos se solapan en su contenido:

- Aquéllos que centran la atención en los prerrequisitos conceptuales necesarios para enseñar estos conceptos complejos o que hacen referencia a la secuenciación de contenidos en base a jerarquías de aprendizaje basadas en el modelo de Gagné (1962).

- Aquéllos otros que enfatizan las aplicaciones de estos conceptos a la hora de realizar cálculos estequiométricos.

- Los que utilizan como nuevas estrategias de enseñanza las analogías o simulaciones por ordenador.

- Y los que giran en torno al pensamiento del profesorado sobre el propio concepto de mol.

A continuación vamos a referirnos a algunos de los trabajos más relevantes que aparecen en la literatura en cada uno de estos apartados.

\section{Dificultades en los prerrequisitos conceptuales o en la secuenciación de contenidos}

Existen abundantes trabajos de investigación que ponen el acento en las dificultades que plantean los prerrequisitos necesarios para su aprendizaje o plantean cuestiones de tipo metodológico. Así, en un estudio de Griffiths y otros (1983), se hace referencia, como prerrequisitos necesarios para el aprendizaje del concepto de $\mathrm{mol}$, a la destreza de saber derivar masas de sustancias a partir del número de partículas presentes y a la necesidad de introducir la masa molar antes de determinar el número real o relativo de partículas presentes en masas de sus- tancias. Por otra parte, Bent (1985) considera prioritario que los estudiantes aprendan a pensar en átomos antes de enseñar el concepto de mol.

Los trabajos de investigación sobre prerrequisitos se extienden a la década de los noventa. En este sentido, el trabajo de Ainley (1991) se refiere a la importancia del concepto de masa atómica relativa y los significados asociados a las fórmulas en las ecuaciones químicas. Asimismo, Hierrezuelo y Montero (1991) hacen referencia a la necesidad de que los estudiantes conozcan la naturaleza corpuscular de la materia y las leyes de la combinación química, y el trabajo de Llorens (1991) refiere la inadecuada distinción entre las magnitudes «cantidad de sustancia» y «masa», así como las deficiencias de interpretación en el significado de las fórmulas químicas. Por otra parte, el trabajo de Izquierdo (1999) muestra la necesidad de aproximarse a la química de las sustancias y de comprender el significado de la «cantidad de sustancia» antes de pasar a la química de los átomos.

Otro tipo de trabajos parten de establecer los prerrequisitos necesarios para realizar una adecuada secuenciación de contenidos teniendo en cuenta las jerarquías de aprendizaje de Gagné (Gower et al., 1977; Griffiths et al., 1983, 1988). Así, Gower y otros (1977) efectuaron un análisis jerárquico en relación con la realización de cálculos básicos de estequiometría utilizando el concepto de $\mathrm{mol}$. De esta forma llegaron a diseñar unos diagramas de flujo para enseñar a realizar cálculos básicos de estequiometría fundamentando su validez en que:

a) representan las etapas esenciales en la resolución de problemas que requieren el uso del mol;

b) muestran la información necesaria y los conceptos requeridos para que el estudiante entienda lo que hace;

c) permiten reconocer las actividades implícitas en un gran número de problemas;

d) pueden ayudarnos a la hora de revisar nuestra propia enseñanza;

e) pueden servir para diagnosticar las razones del fracaso al resolver los problemas.

En otro trabajo de investigación en la línea de la teoría del procesado de la información, Lazonby y otros (1982) encontraron que la ordenación y la redacción del enunciado de una cuestión son importantes para que sea captada y entendida por los estudiantes. También encontraron que había falta de comprensión en lo que respecta a la interpretación molar de los subíndices en las fórmulas químicas y los coeficientes de las ecuaciones. En definitiva, llegaron a la idea de que quizá el mol en sí mismo no es algo confuso y que es el modo o el camino utilizado para llegar a él, lo que confunde a los estudiantes. En un trabajo posterior realizado con una amplísima muestra de 2.695 estudiantes de 15 a 16 años de edad, Lazonby y otros (1985) mostraban que una secuenciación adecuada de las cuestiones que se plantean a los 
estudiantes facilita su comprensión, llegando a la conclusión de que el orden a seguir en la enseñanza debería determinarse a partir de las dificultades relativas de las operaciones tal y como son percibidas por los estudiantes. En este sentido, McCullough (1990) propone diagramas para facilitar el aspecto operativo de la relación entre masas, «cantidades de sustancia» y número de partículas.

Ben-Zvi y otros (1988) investigaron la simbología utilizada, por estudiantes de 15 años en los niveles macroscópico, atómico-molecular y poliatómico, y encontraron que muchos de ellos tenían una representación mental distorsionada del modelo atómico. Se apuntaron varias características que lo hacen difícil: la inclusión de conceptos abstractos (como átomo y molécula), así como la expresión simbólica con múltiples interpretaciones posibles.

Sin embargo, Chiappetta y McBride (1980) encontraron que no había un remedio general para enseñar el concepto de $\mathrm{mol}$ en cursos introductorios a estudiantes que no tenían éxito en el aprendizaje del mismo. Así mismo, en una investigación de Griffiths y otros (1988) sobre cálculos estequiométricos utilizan el concepto de mol y aplican la teoría de aprendizaje de Gagné. El objetivo es averiguar si mejora (respecto a otros estudiantes no tratados) el rendimiento de los estudiantes a los que se proporciona un tratamiento que incluye referencias a sus propios errores conceptuales. Los resultados obtenidos no son muy alentadores en cuanto a las diferencias en el rendimiento obtenido por los estudiantes antes y después del tratamiento efectuado.

\section{Utilización de analogías y nuevas tecnologías como estrategias didácticas}

En torno a las dificultades anteriormente apuntadas, también abundan en la literatura didáctica las referencias a la necesidad de utilizar analogías familiares, mediante las que se pretende facilitar el aprendizaje del concepto de mol. Algunas de dichas analogías hacen referencia a determinados prerrequisitos del concepto de mol. En este sentido, para enseñar el concepto de peso fórmula relativo de una sustancia, Felty (1985) propone como situación analógica la preparación de una ensalada de frutas con igual número de uvas y de cerezas: «[...] vas a la tienda y pides igual número de uvas y de cerezas. El tendero contesta: Lo siento, las vendemos por peso, ¿cuánto peso desea de cada una?». El razonamiento sería: «si comprara el mismo número de libras de cada una, solamente tendría el mismo número de uvas y de cerezas en el caso de que las uvas y las cerezas (cada una) tuvieran el mismo peso (idéntico), pero probablemente no es el caso. Necesito saber sus pesos relativos.»

Por otra parte, con el fin de enseñar el concepto de masa atómica media de un elemento del que se conocen las masas atómicas y el porcentaje de abundancia de sus dos isótopos, Last y Webb (1993) utilizan analogías monetarias planteando a los estudiantes que imaginen una situación en la que diez estudiantes trabajan a tiempo parcial: siete de ellos lo hacen en un trabajo en el cual cada uno gana cierta cantidad por semana, y los otros tres tienen otro tipo de trabajo en el cual cada uno gana otra cantidad diferente por semana. El problema es averiguar la ganancia media de los estudiantes.

Con objeto de facilitar el aprendizaje del concepto de masa atómica relativa, otros autores plantean analogías utilizando conjuntos de monedas. En opinión de Henson y Stumbles (1979), la elección de este tipo de analogías se basa en la familiaridad que tiene para los estudiantes el hecho de que, en los bancos, en lugar de contar monedas se efectúan pesadas. Si se asocia a cada tipo de moneda una masa determinada cuyo valor sea proporcional (por ejemplo, si la masa de una moneda de 2 peniques es doble que la de 1 penique, y ésta es doble que la de medio penique), pueden calcularse las masas relativas de las monedas y establecer así las correspondientes relaciones con las masas atómicas relativas. Myers (1989) plantea una situación similar utilizando peniques y centavos, mientras que De Berg (1986b) propone utilizar piezas de cartulina de masas diferentes $(0,1 \mathrm{~g}$, $0,2 \mathrm{~g}, 0,5 \mathrm{~g}$ y $0,7 \mathrm{~g}$, respectivamente).

En relación con el concepto de masa atómica relativa, Arce de Sanabia (1993) propone utilizar como situación analógica clips de diferente tamaño metidos en cajas de cerillas. Para ello se llenan varias cajas de cerillas (dos o tres) con un número igual de clips de diferente tamaño, que los estudiantes no pueden observar directamente. A continuación se anima a los estudiantes a pensar sobre la posibilidad de determinar por pesada el número de clips que contiene cada caja. Una vez determinada la masa de las cajas vacías se averigua, por diferencia, la masa de los clips contenidos en cada caja. A partir de aquí se tiene en cuenta que la masa total en cada caja es igual a la masa de cada clip multiplicada por el número que contiene, por tanto, puede averiguarse la masa de cada tipo de clip respecto al más ligero de ellos (masa relativa). En relación con el mismo concepto de masa atómica relativa, otros autores proponen analogías con diferentes tipos de animales: cerdos, perros y pollos (Chamberlain et al., 1991; Fortman, 1993).

También se encuentran propuestas para facilitar la comprensión de determinados aspectos relacionados con aplicaciones del concepto de mol. Por ejemplo, con objeto de superar las dificultades de los estudiantes al utilizar fracciones molares en los cálculos de la concentración de una disolución expresada como fracción molar de soluto, De Lorenzo (1980) propone, como aproximación familiar, el cálculo previo de la fracción de estudiantes femeninos en una clase formada por chicos y chicas. Con el fin de facilitar la utilización de proporciones de moles de sustancias que intervienen en una reacción, Fortman (1994) propone la utilización de analogías que centren la atención de los estudiantes en el manejo de número de entidades, aunque éstas puedan ser expresadas en términos de masa o de volumen.

Son muy abundantes las analogías propuestas para facilitar el aprendizaje del concepto de mol y cómo los 
estudiantes pueden familiarizarse con el número de Avogadro. En este sentido, Fulkrod (1981) propone el cálculo del volumen ocupado por el número de Avogadro de gotas de agua, para lo cual se parte de asumir que 20 gotas de agua ocupan un volumen de $1 \mathrm{~cm}^{3}$. Para hacerse una idea del tamaño de átomos y moléculas y la magnitud del número de Avogadro, Alexander y otros, (1984) proponen varias situaciones analógicas: el cálculo del crecimiento de la barba por segundo, tomando como referencia un centímetro por mes. A partir de aquí deducen un crecimiento de 3,9 nm por segundo y lo comparan con el diámetro de un átomo de carbono (10 veces). Para estimar el tamaño de las moléculas, comparan el hipotético tamaño que tendría una molécula de agua (como un grano de sal) respecto a una hormiga en el caso que ésta pareciera tener una altura de una milla. Para ilustrar la magnitud del número de Avogadro lo comparan con el volumen del océano pacífico expresado en milílitros $\left(7,10^{23}\right)$.

Con objeto de acostumbrar a los estudiantes en la comprensión de conceptos relacionados con el mol, Gabel y Sherwood (1984) proponen la utilización de tareas familiares con naranjas y con gránulos de azúcar. Conocidos el número de las entidades respectivas, así como el peso y el volumen de sendos sacos de naranjas y de azúcar, se solicita de los estudiantes el cálculo de cantidades en diferentes supuestos.

Son muy abundantes las analogías propuestas para familiarizarse con el número de Avogadro basándose en calcular diferentes cantidades de entidades como:

- cálculo de la masa de átomos de hidrógeno contenidos en el volumen terrestre (Todd, 1985);

- cálculos de: $a$ ) el dinero al repartir un mol de dólares de forma equitativa entre los habitantes de la tierra o de la superficie ocupada por un mol de hormigas, suponiendo que se conocen la masa media de una hormiga, la superficie de un hormiguero, la masa y la superficie terrestre; $b$ ) la masa y el volumen que representan un mol de granos de arena a partir de la masa de un grano de arena, la densidad de la arena, la masa y la superficie terrestre (Van Lubeck, 1989);

- cálculo del dinero sobrante al gastar 106 dólares cada segundo, desde el momento de la formación de la tierra, presuponiendo un capital inicial de un mol de dólares (Tannenbaum, 1990);

- cálculo del volumen ocupado por un mol de canicas, de perdigones, de granos de arena, etc. (Hoyt, 1992);

- cálculo del volumen que ocupan un mol de pequeños caramelos (Merlo y Turner, 1993);

- utilización del mol como equivalente a una moneda química (Bonneau, 1994);

- utilización de envases cilíndricos coloreados para visualizar las leyes de los volúmenes de combinación (Gay-Lussac) y de Avogadro (Bouma, 1986).

Con objeto de ver la necesidad de contar partículas por pesada en química, Dominic (1996) sugiere averiguar el número de caramelos contenidos en una jarra sin contarlos directamente. Por otra parte, Poskozim y otros (1986) efectuaron una revisión sobre las analogías utilizadas para introducir el número de Avogadro en los libros de texto, encontrando que éstas se basan en el cálculo del volumen ocupado por pequeños objetos, el tiempo necesario para contarlos, etc.

Rowell y Dawson (1980) idearon una estrategia de instrucción de seis semanas de duración (sin emplear grupo de control) en la que utilizaban como analogía las monedas de uso corriente en el entorno de los estudiantes. Mostraron que aproximadamente, en la mitad de estudiantes de secundaria tratados (15 años), la estrategia utilizada era parcialmente efectiva para la enseñanza del concepto de mol.

Así mismo, Friedel y otros (1990), en la continuación de un estudio inicial sobre dificultades, mostraron que la utilización de analogías en la enseñanza de la química puede resultar fructífera si se cumplen varias condiciones:

- la analogía debe ser inteligible;

- deben verse las relaciones entre la situación analógica familiar al alumno y la situación química en la que se contextualiza el concepto;

- deben poder transferirse las soluciones dadas a la situación analógica familiar al contexto del problema de química que se quiere resolver;

- deben utilizarse durante un largo período de tiempo.

En relación con las nuevas tecnologías, empiezan a aparecer en la bibliografía trabajos que emplean simulaciones en ordenador. Así, Yalçinalp y otros (1995) estudiaron la influencia de un diseño de enseñanza asistida por ordenador sobre un centenar de estudiantes de educación secundaria y encontraron mejoras significativas en el aprendizaje del concepto de mol y las fórmulas químicas, además de mejorar las actitudes hacia la química. Toloudis (1996) ha elaborado un sencillo programa en BASIC para mostrar la enorme magnitud del número de Avogadro midiendo el tiempo necesario para contar el número de entidades contenidas en un mol. Otros autores, Dori y Hameiri (1998), plantean la aplicación del análisis multidimensional a la resolución de problemas cuantitativos de química (cálculo de la masa o el número de átomos contenidos en cierta cantidad de sustancia, etc.), para lo cual proponen la resolución de ejercicios de opción múltiple utilizando el ordenador de forma interactiva a la hora de responder y ofreciendo la posibilidad de obtener información suplementaria (en relación con aspectos de ciencia y sociedad) para los estudiantes que resuelven correctamente los ejercicios.

\section{Estrategias de aplicación del concepto de mol en los cálculos estequiométricos}

Frazer y Servant (1987), en un trabajo posterior a los anteriormente citados, al comentar con detalle los pobres resultados obtenidos por los estudiantes en su pri- 
mer trabajo (1986a) -en un $79 \%$ de las equivocaciones totales, en lo que respecta a magnitudes confundidas, aparece la «cantidad de sustancia»-, se refieren a la considerable confusión originada por los intentos de introducir en el aula la terminología moderna en relación con la «cantidad de sustancia» y el mol, así como a la necesidad de métodos de cálculo que permitan reforzar la comprensión de los estudiantes.

De Berg (1986b) propone una estrategia para realizar cálculos estequiométricos con el mol y comenta la presentación inadecuada de este concepto en los libros de texto. En el análisis del contenido de libros de texto respecto a cómo se introduce el concepto de $m o l$, de Berg (1986a) encontró que existía una variedad de aproximaciones para llegar a este concepto. Así mismo, dicho autor llegó a la conclusión de que no podía establecerse a priori que una determinada jerarquía conceptual fuera más válida que otra, y que esto sólo podría efectuarse a través de la puesta en práctica con los estudiantes.

Packer (1988), tomando como referencia el trabajo de Frazer y Servant (1986a) antes citado, critica como inadecuado el método utilizado por los profesores de química para ofrecer a los estudiantes la comprensión y las técnicas necesarias para resolver problemas de estequiometría, y apunta la necesidad de superar estos deficientes métodos de enseñanza para que los estudiantes entiendan lo que hacen en las clases.

Por otra parte, un amplio número de investigaciones (Lee R.E., 1982; Woods, 1982; Gorin, 1987; Hoppé, 1990) considera que las dificultades de los estudiantes son atribuibles a una enseñanza inicial deficiente o a las inadecuadas explicaciones del profesorado. Así mismo, se argumenta que no hay tiempo para introducir el concepto de mol en el extenso programa de los cursos de iniciación a la química (Bent, 1987).

\section{CONCLUSIONES}

La revisión bibliográfica realizada pone de manifiesto una clara discrepancia entre lo asumido por la comunidad científica (Mills et al., 1993) y el pensamiento del profesorado, reflejado en la información contenida en los libros de texto, respecto al significado y al papel relevante de la magnitud «cantidad de sustancia» y del mol. En concreto, este desacuerdo se refleja en la enseñanza en los aspectos siguientes:

a) El concepto de cantidad de sustancia no es introducido en la gran mayoría de los programas de enseñanza habitual de la química. En este sentido, se suele identificar cantidad de sustancia con masa o con número de entidades elementales, desconociendo su significado actual como magnitud que sirve para contar partículas.

b) Los conceptos de cantidad de sustancia y de mol se confunden con conceptos incluidos en la teoría atómicomolecular tales como masa molar, constante de Avogadro... c) Dificultades de secuenciación de los contenidos al introducir el concepto de mol e inadecuación de las metodologías de enseñanza utilizadas habitualmente.

De acuerdo con Gabel y Bunce (1994), creemos que las graves deficiencias detectadas en la enseñanza pueden ser, en parte, responsables de las dificultades de aprendizaje apuntadas en la bibliografía. Tal como se pone de manifiesto en la revisión realizada, los estudiantes tienen dificultades en manejar de modo significativo los conceptos de cantidad de sustancia y de mol. Así mismo, en la resolución de problemas, los estudiantes no suelen utilizar estrategias basadas en la determinación de «cantidades de sustancia». En la revisión bibliográfica realizada, desde el punto de vista de aprendizaje, se han apuntado los siguientes aspectos relevantes:

a) Los estudiantes carecen de una concepción científica del mol.

b) La gran mayoría de los estudiantes identifica el mol con una masa, con un volumen o como un número de entidades elementales.

c) Los estudiantes desconocen el significado de la magnitud «cantidad de sustancia», evitan su manejo significativo y no identifican el mol como su unidad.

d) Los estudiantes confunden frecuentemente el nivel macroscópico de representación (masa molar) con el microscópico (masa atómica y masa molecular).

e) Los estudiantes suelen identificar la proporción de moléculas con la proporción de masas y la proporción de masas con la proporción de masas molares.

Así mismo, la revisión realizada resalta que las concepciones actuales respecto a la magnitud «cantidad de sustancia» y su unidad, el mol, son el resultado de un largo proceso de investigación en torno al problema de la determinación de cantidades en las reacciones químicas dentro de un marco teórico atomista. En este sentido se distingue entre el contexto original de indagación de Ostwald y el contexto actual, recomendándose diferenciar claramente entre cantidad de sustancia, masa, volumen y número de entidades elementales.

De acuerdo con la recopilación de dificultades de aprendizaje realizada, podemos apuntar que el verdadero problema en torno a la «cantidad de sustancia» es que se trata de una magnitud macroscópica que se relaciona directamente con el mundo microscópico de las sustancias (átomos y moléculas) y, para poder relacionar estos dos niveles de representación, hay que interiorizarlos previamente; esto es, hay que relacionar las definiciones macroscópicas de sustancia y reacción química con el nivel microscópico atomista.

Es preciso resaltar la relevancia y la plena vigencia del problema didáctico que plantea la comprensión de los conceptos de cantidad de sustancia y de mol, debido a las repercusiones que tiene a nivel de enseñanza y 
aprendizaje de la química. Tal como se ha mostrado en la revisión que aquí presentamos, este problema no se limita a los errores de los estudiantes en la comprensión

\section{REFERENCIAS BIBLIOGRÁFICAS}

AINLEY, D. (1991). Mole catchers? Education in Chemistry, 28(18), pp. 18-19.

ALEXANDER, M.D., EWING, G.J. y ABBOT, F.T. (1984). Analogies that indicate the size of atoms and molecules and the magnitude of Avogadro's number. Journal of Chemical Education, 61(7), p. 591.

ALLSOP, R.T. (1977). The place and importance of the mole in school chemistry courses. Physics Education, 12, pp. 285288.

ARCE DE SANABIA, J. (1993). Relative atomic mass and the mole: a concrete analogy to help students understand these abstract concepts. Journal of Chemical Education, 70(3), pp. 233-234.

AZCONA, R. (1997). Origen y evolución de los conceptos de cantidad de sustancia y mol. Implicaciones en la enseñanza de la química. XII Cursos sobre aspectos didácticos en la enseñanza secundaria: química. Colección Educación Abierta ICE: Zaragoza.

AZCONA, R. (2001). Análisis crítico de la enseñanza-aprendizaje de los conceptos de cantidad de sustancia y de mol. Una alternativa didáctica basada en el aprendizaje como investigación, en Investigaciones en didáctica de las ciencias experimentales basadas en elmodelo de enseñanza-aprendizaje como investigación orientada. Guisasola, J. y Pérez de Eulate, L. (eds.). Bilbao: Universidad del País Vasco.

BENT, H.A. (1985). Should the mole concept be X-rated? Journal of Chemical Education, 62(1), p. 59.

BENT, H.A. (1987). Should we «teach the mole»? Journal of Chemical Education, 64(3), p. 192.

BEN-ZVI, R., EYLON, B. y SILBERSTEIN, J. (1988). Theories, principles and laws. Education in Chemistry, 25(3), pp. 8992.

BIEBER, T. (1961). Letter to the Editor. Journal of Chemical Education, 38(11), p. 254.

BONNEAU, M.C. (1994). The mole buck. Journal of Chemical Education, 71(4), p. 286.

BOUMA, J. (1986). Gas cans and gas cubes: visualizing Avogadro's law. Journal of Chemical Education, 63(7), pp. 586-587.

BROWN, B.S. (1991). A mole mnemonic. Journal of Chemical Education, 68(12), p. 1039.

CAAMAÑO, A. (1983). La gramática del lenguaje científico (II). Magnitudes físicas y químicas. Cuadernos de Pedagogía, 98 , pp. 64-67. y la utilización de estos conceptos, sino que también tiene que ver con las ideas del profesorado y con las metodologías utilizadas en la enseñanza de los mismos.
CAAMAÑO, A., MAYOS, C., MAESTRE, G. y VENTURA, T. (1983). Consideraciones sobre algunos errores conceptuales en el aprendizaje de la química en el bachillerato. Enseñanza de las Ciencias, 1(3), pp. 198-200.

CERVELlATI, R., MONTUSCHI, A., PERUGINI, D., GRIMELLINI-TOMASINI, N. y PECORINI BALANDI, B., 1982. Investigation of secondary school students' understanding of the mole concept in Italy. Journal of Chemical Education, 59(10), pp. 852-856.

CHAMBERLAIN, D., RITCHIE, A. y STRATTON, J. (1991). El concepto de mol. Guía del profesor. TV Ontario. Madrid: International Education and Training Enterprises.

CHIAPPETTA, E.L. y McBRIDE, J.W. (1980). Exploring the effects of general remediation on ninth-graders' achievement of the mole concept. Science Education, 64(5), pp. 609-614.

CLAYTON, D.G. (1981). The variable mole and moleage. Education in Chemistry, 18(6), p. 164.

CLAYTON, D. (1982). The elusive S.I. mole. Education in Chemistry, 19(4), p. 102.

CLAYTON, D.G. (1983). The mole. Education in Chemistry, 20(2), p. 36.

COHEN, I. (1961a). Letter to the Editor. Journal of Chemical Education, 38(11), p. 554.

COHEN, I. (1961b). Moles and equivalents: quantities of matter. Journal of Chemical Education, 38(11), pp. 555-556.

COPLEY, G.N. (1961). The mole in Quantitative Chemistry. Journal of Chemical Education, 37(11), pp. 551-553.

DE BERG, K.C. (1986a). Fundamental calculations with the mole. The Australian Science Teachers Journal, 32(1), pp. 29-36.

DE BERG, K.C. (1986b). Text book analysis of the mole and its underlying concepts. A teaching-learning perspective. The Australian Science Teachers Journal, 32(4), pp. 33-43.

DE LORENZO, R. (1980). Mole fraction analogies. Journal of Chemical Education, 57(10), p. 733.

DIERKS, W. (1981). Teaching the mole. European Journal of Science Education, 3(2), pp. 145-148.

DOMINIC, S. (1996). What's a mole for? Journal of Chemical Education, 73(4), p. 309.

DOIRI, Y.J. y HAMEIRI, M. (1998). The «Mole Environment» studyware: applying multidimensional analysis to quantitative chemistry problems. International Journal of Science Education, 20(3), pp. 317-333. 
DRIVER, R. (1986). Psicología cognoscitiva y esquemas conceptuales de los alumnos. Enseñanza de las Ciencias, 4(1), pp. 3-15.

DUNCAN, I.M. y JOHNSTONE, A.H. (1973). The mole concept. Education in Chemistry, 10, pp. 213-214.

ELSWORTH, J.F. (1990). Concentrating on change. Education in Chemistry, 27(3), p. 100.

FELTY, W.L. (1985). Gram formula weights and fruit salad. Journal of Chemical Education, 62(1), p. 61.

FERNÁNDEZ, M.L. (1989). Errores en el concepto de mol. Apuntes de Educación. Naturaleza y Matemáticas, 35, pp. 6-8.

FORBES, R.G. (1976). A fundamental proposal concerning the mole. Education in Chemistry, 13, p. 92.

FORBES, R.G. (1977). ... and interpreting it. Letter to the Editor. Education in Chemistry, 14, p. 124.

FORBES, R.G. (1978a). More confusion over the Avogadro constant. Physics Education, 13, pp. 5-6.

FORBES, R.G. (1978b). Amount of substance: an alternative proposal. Physics Education, 13, pp. 269-272.

FORBES, R.G. (1982). The seventh S.I. quantity. Education in Chemistry, 19(4), p. 102.

FORBES, R.G. (1991). The physicists' amount too. The Science School Review, 73(263), p.133.

FORTMAN, J.J. (1993). Pictorical analogies IV: Relative atomic weights. Journal of Chemical Education, 70(3), pp. 235-236.

FORTMAN, J.J. (1994). Stoichiometry calculations. Journal of Chemical Education, 71(7), pp. 571-572.

FOY, J.R. (1961). Letter to the Editor. Journal of Chemical Education, 38(11), p. 554.

FRAZER, M.J.y SERVANT, D. (1986a). Aspects of stoichiometry titration calculations. Education in Chemistry, 23(2), pp. 54-56.

FRAZER, M.J. y SERVANT, D. (1986b). Aspects of stoichiometry. A wider view? Education in Chemistry, 23(5), pp. 138-140.

FRAZER, M.J y SERVANT, D.M. (1987). Aspects of stoichiometry-where do students go wrong? Education in Chemistry, 24(3), pp. 73-75.

FRIEDEL, A., GABEL, D.L. y SAMUEL, J. (1990). Using analogs for Chemistry problem solving: does it increase understanding? School Science and Mathematics, 90(8), pp. 674-682.

FULKROD, J.E. (1981). How big is Avogadro's number (or how small are atoms, molecules and ions)? Journal of Chemical Education, 58(6), p. 508.

FURIÓ, C., AZCONA, R., GUISASOLA, G. y MUJIKA, E. (1993). Concepciones de los estudiantes sobre una magnitud «olvidada» en la enseñanza de la química: la cantidad de sustancia. Enseñanza de las Ciencias, 11(2), pp. 107-114.

FURIÓ, C., AZCONA, R. y GUISASOLA, J. (1999). Dificultades conceptuales y epistemológicas del profesorado en la enseñanza de los conceptos de cantidad de sustancia y de mol. Enseñanza de las Ciencias, 17(3), pp. 359-376.

GABEL, D.L. y BUNCE, D.M. (1994). Handbook of research on science teaching and learning. A Project of the National
Science Teachers Association. Research on problem solving: Chemistry. Nueva York: MacMillan Publishing Company.

GABEL, D. y SHERWOOD, R.D. (1984). Analyzing difficulties with mole-concept task by using familiar analog tasks. Journal of Research in Science Teaching, 21(8), pp. 843851 .

GAGNÉ, R.M. (1962). The acquisition of knowledge. Psychological Review, 69(4), pp. 355-365.

GARCÍA, J.P., PIZARRO, A., PERERA, F., MARTÍN, M. MYORDF y BACAS, P. (1990). Ideas de los alumnos acerca del mol. Estudio curricular. Enseñanza de las Ciencias, 8(2), pp. 111-119.

GOODSTEIN, M. y HOWE, A. (1978). The use of concrete methods in secondary chemistry instruction. Journal of Research in Science Teaching, 15(5), pp. 361-366.

GORIN, G. (1982). «Chemical amount» or «Chemiance»: proposed names for the quantity measured in mole units. Journal of Chemical Education, 59(6), p. 508.

GORIN, G. (1983). What do we measure in moles? Journal of Chemical Education, 60(10), p. 782.

GORIN, G. (1984). The unit gram/mole and its use in the description of molar mass. Journal of Chemical Education, 61(12), p. 1045.

GORIN, G. (1985). The definition and symbols for the quantity called «molarity» or «concentration» and for the S.I. units of this quantity. Journal of Chemical Education, 62(9), pp. 741.

GORIN, G. (1987). Should we «teach the mole»? Journal of Chemical Education, 64(3), p. 192.

GORIN, G. (1994). Mole and chemical amount. A discussion of the fundamental measurements of Chemistry. Journal of Chemical Education, 71(2), pp. 114-116.

GOWER, D.M., DANIELS, D.J. y LLOYD, G. (1977). The mole concept. The School Science Review, 58(205), pp. 658676.

GRIFFITHS, A.K., KASS, H. y CORNISH, A.G. (1983). Validation of a learning hierarchy for the mole concept. Journal of Research in Science Teaching, 20(7), pp. 639654.

GRIFFITHS, A.K., THOMEY, K., COOKE, B. y NORMORE, G. (1988). Remediation of student-specific misconceptions relating to three science concepts. Journal of Research in Science Teaching, 25(9), pp. 709-719.

GUGGENHEIM, E.A. (1961). The mole and related quantities. Journal of Chemical Education, 30(2), pp. 86-87.

HAWTHORNE, R.M. (1973). The mole and Avogadro's number. Journal of Chemical Education, 50(4), pp. 282-284.

HENSON, R. y STUMBLES, A. (1979). Modern mathematics and the mole. Education in Chemistry, 16(1), pp. 10-11.

HERRON, J.D. (1975). Piaget for chemists. Journal of Chemical Education, 52(3), pp. 146-150.

HIERREZUELO, J. y MONTERO, A. (1991). La ciencia de los alumnos: su utilización en la didáctica de la física y química. Vélez-Málaga: Elzevi.

HOPPÉ, J. (1990). The mole digs deeper... Education in Chemistry, 27(5), p. 129.

HOPPÉ, J. (1991). Chemical amount or chemount. The Science School Review, 73(263), pp. 132-133. 
HOYT, W. (1992). A mole of salt crystals-or how big is the Avogadro's number? Journal of Chemical Education, 69(6), p. 496 .

HUDSON, M.J. (1976). Introducing the mole. Education in Chemistry, 13(4), pp. 110-114.

IZQUIERDO, M. (1999). La emergencia de la explicación cuantitativa en química. VII Congreso de la Sociedad Española de Historia de las Ciencias y de las Técnicas. Estudios de Historia das Ciencias edas Técnicas. Pontevedra: Diputación Provincial. Servicio de Publicaciones.

JOHNSTONE, A.H. y EL-BANNA, H. (1986). Capacities, demands and processes-a predictive model for science education. Education in Chemistry, 23, pp. 80-84.

KOHMAN, T.P. (1987). Molar and equivalent amounts and concentrations. Journal of Chemical Education, 64(3), p. 246.

KOLB, D. (1978). The mole. Journal of Chemical Education. 55(1), pp. 728-732.

KRISHNAN, S.R. y HOWE, A.C. (1994). The mole concept developing an instrument to assess conceptual understanding. Journal of Chemical Education, 71(8), pp. 653-655.

LAST, A.M. y WEBB, M.J. (1993). Using monetary analogies to teach average atomic mass. Journal of Chemical Education, 70(3), pp. 234-235.

LAZONBY, J.N., MORRIS, J.E. y WADDINGTON, D.J. (1982). The muddlesome mole. Education in Chemistry, 19(4), pp. 109-111.

LAZONBY, J.N., MORRIS, J.E. y WADDINGTON, D.J. (1985). The mole: questioning format can make a difference. Journal of Chemical Education, 62(1), pp. 60-61.

LEE, R.E. (1982). The constant mole. Education in Chemistry, 19(1), pp. 6-7.

LEE, S. (1961). A redefinition of «mole». Journal of Chemical Education, 38(11), pp. 549-551.

LLORENS, J.A. (1991). Comenzando a aprender química. Ideas para el diseño curricular. Madrid: Aprendizaje-Visor.

MAcDONALD, J.J. (1984). The mole: how should it be taught? The School Science Review, 65(232), pp. 486-497.

MASSON, M.R. (1993). ...or not to be. Letter to the Editor. Education in Chemistry, 30(1), p. 11.

McCULLOUGH, T. (1990). Avogadro's number, moles and molecules. Journal of Chemical Education, 67(9), p. 783.

McGLASHAN, M.L. (1977). Amount of substance and the mole. Physics Education, 12, pp. 276-278.

McMANUS, F.R. (1982). Amount of substance. Education in Chemistry, 19(1), p. 7.

McMANUS, F.R. (1983). The abstract mole. Education in Chemistry, 20(1), p. 6.

MERLO, C. y TURNER, K. (1993). A mole of M and M's. Journal of Chemical Education, 70(6), p. 453.

MILLS, I.M. (1989). The choice of names and symbols for quantities in Chemistry. Journal of Chemical Education, 66(11), pp. 887-889.

MILLS, I.M. (1990). ... and deeper. Education in Chemistry, $27(5)$, p. 129.
MILLS, I.M., CVITAS, T., HOMANN, K, KALLAY, N. y KUCHITSU, K. (1993). IUPAC Quantities, units and symbols in physical chemistry. Oxford: Blackwell.

MYERS, R.T. (1989). Moles, pennies and nickels. Journal of Chemical Education, 66(3), p. 249.

NELSON, P.G. (1989). Stoichiometry. Education in Chemistry, 26(1), p. 8.

NELSON,P.G.(1991). The elusive mole. Education in Chemistry, 28(4), pp. 103-104.

NELSON. P.G. (1994). Introducing ...atoms and molecules. Education in Chemistry, 31(1), pp. 20-21.

NIAZ, M. (1985). Evaluation of formal operational reasoning by Venezuelan freshmen students. Research in Science and Technological Education, 3(1), pp. 43-50.

NIAZ, M. (1987). Estilo cognoscitivo y su importancia para la enseñanza de la ciencia. Enseñanza de las Ciencias, 5(2), pp. 97-104.

NIAZ, M. (1988). The information-processing demand of chemistry problems and its relation to Pascual-Leone's functional M-capacity. International Journal of Science Education, 10(2), pp. 231-238.

NIAZ, M. (1989). Relation between Pascual-Leone's structural and functional M-space and its effect on problem solving in Chemistry. International Journal of Science Education, 11(1), pp. 93-99.

NOVICK, S. y MENIS, J. (1976). A Study of student perceptions of the mole concept. Journal of Chemical Education, 53(11), pp. 720-722.

PACKER, J.E. (1988). Difficulties with stoichiometry. Education in Chemistry, 25(3), pp. 92-95.

PASCUAL-LEONE, J. y GOODMAN, D. (1979). Intelligence and experience. Instructional Science, 8, pp. 301-367.

POSZOKIM, P.S., WAZORICK, J.W., TIEMPETPALSAL, P y POSZOKIM, J.A. (1986). Analogies for Avogadro's number. Journal of Chemical Education, 63(2), pp. 125-126.

RAMETTE, R.W. (1988). The mole concept is useful. Journal of Chemical Education, 65(4), p. 376.

RETHERFORD, K.L. (1978). Avogadro's number. Journal of Chemical Education, 55(5), p. 334.

ROCHA-FILHO, R.C. (1990). A proposition about the quantity of which mole is the S.I. unit. Journal of Chemical Education, 67(2), pp. 139-140.

ROWELL, J.A. y DAWSON, C.J. (1980). Mountain or mole hill: can cognitive psychology reduce the dimensions of conceptual problems in classroom practice? Science Education, 64(5), pp. 693-708.

SCHMIDT, H.J. (1990). Secondary School students' strategies in stoichiometry. International Journal of Science Education, 12(4), pp. 457-471.

SCHMIDT, H.J. (1994). Stoichiometry problem solving in high school Chemistry. International Journal of Science Education, 16(2), pp. 191-200.

SHAYER, M. y ADEY, P. (1984). La ciencia de enseñar ciencias. Madrid: Narcea.

SMITH, C.G. (1984). The abstract mole. Education in Chemistry, 21(4), p. 109 
SPURGIN, B. (1992). Amount of substance. The School Science Review, 73(265), pp. 151-152.

STAVER, J.R. y LUMPE, A.T. (1993). A content analysis of the presentation of the mole conception in Chemistry texbooks. Journal of Research in Science Teaching, 30(4), pp. 321337.

STAVER, J.R. y LUMPE, A.T. (1995). Two Investigations of students» understanding of the mole concept and its use in problem solving. Journal of Research in Science Teaching, 32(2), pp. 177-193.

STEINER, R.P. (1986). Teaching stoichiometry. Journal of Chemical Education, 63(12), p. 1048.

STRÖMDAHL, H., TULBERG, A. y LYBECK, L. (1994). The qualitatively different conceptions of 1 mol. International Journal of Science Education, 16(1), pp. 17-26.

TANNENBAUM, I.R. (1990). How large is a mole? Journal of Chemical Education, 67(6), p. 481.

TEN HOOR, M.J. (1993). Molar mass. Education in Chemistry, 30(3), p. 75.

TODD, D. (1985). Five Avogadro's number problems. Journal of Chemical Education, 62(1), p. 76.

TOLOUDIS, M. (1996). The size of a mole. Journal of Chemical Education, 73(4), p. 348.
TULLBERG, A., STRÖMDAHL, H. y LYBECK, L. (1994). Students' conceptions of $1 \mathrm{~mol}$ and educators' conceptions of how they teach «the mole». International Journal of Science Education, 16(2), pp. 145-156.

TYKODI, R.J. (1983). What do we measure in moles? Journal of Chemical Education, 60(10), p. 782.

VAN LUBECK, H. (1989). How to visualize Avogadro's number. Journal of Chemical Education, 66(9), p. 762.

VERDÚ, J. (1993). Sobre los errores en el uso del concepto de mol y de las magnitudes relacionadas. Revista Española de Física, 7(1), pp. 54-56.

VIGOTSKY, L.S. (1989). El desarrollo de los procesos psicológicos. Barcelona: Crítica.

VINCENT, A. (1981). Volumetric concepts-student difficulties. Education in Chemistry, 18(4), pp. 114-115.

WOODS, G.T. (1982). It's that mole again. Education in Chemistry, 19(6), p. 165.

WOODS, G.T. (1991). The chemist's amount. The Science School Review, 72(261), pp. 150-151.

YALÇINALP, S., GEBAN, Ö. y ÖZKAN,Í. (1995). Effectiveness of Using Computer-Assisted Supplementary Instruction for Teaching the Mole Concept. Journal of Research in Science Teaching, 32(10), pp. 1083-1095.

[Artículo recibido en diciembre de 2000 y aceptado en diciembre de 2001.] 